\title{
Réacteurs thermochimiques solaires. I. - Mise au point et caractérisation d'un réacteur à lit brassé
}

\author{
J. M. Badie, R. Foro, D. Hernandez et A. Rouanet \\ Laboratoire des Ultra-Réfractaires, C.N.R.S., BP no 5, Odeillo, 66120 Font-Romeu, France
}

(Reçu le 11 juin 1982, révisé le 20 octobre, accepté le 27 octobre 1982)

\begin{abstract}
Résumé. - En vue de la valorisation de l'énergie solaire par voie thermique, nous avons réalisé un réacteur à lit brassé tournant associé à un four solaire $(2 \mathrm{~kW})$ à forte concentration $(16000)$. Ce réacteur permet des traitements continus dans l'air et jusqu'à $11^{\circ} \mathrm{C}$ (décarbonatation, déshydratation...) de poudres $(100-300 \mu \mathrm{m})$. L'enceinte réactionnelle, constituée par un tube en acier inoxydable (NS 30) de petit diamètre $(4,2$ ou $5,2 \mathrm{~cm})$ et de $12 \mathrm{~cm}$ de longueur, sert à la fois d'absorbeur de l'énergie solaire concentrée et de paroi de transfert vers la charge. Le profil de température sur le cylindre dépend directement de la distribution de l'éclairement solaire incident dans la cavité. La différence de température n'excède pas $100^{\circ} \mathrm{C}$ pour une température moyenne de $1000^{\circ} \mathrm{C}$. Le temps de séjour moyen des particules dans le réacteur est de 1 à $2 \mathrm{~min}$. pour des débits massiques de 5 à $25 \mathrm{~g} . \mathrm{min}^{-1}$ et des vitesses de rotation de 2 à 8 tours par minute.
\end{abstract}

\begin{abstract}
A rotary kiln associated with a high concentration (16000) solar furnace ( $2 \mathrm{~kW}$ power) was built in view to valorize solar energy using thermal means. The reactor allows a continuous $100-300 \mu \mathrm{m}$ powders processing in air up to $11^{\circ} \mathrm{C}$ (decarbonation, dehydration...). The reactive vessel, made of a stainless steel (NS 30) tube (4.2 or $5.2 \mathrm{~cm}$ diameter and $12 \mathrm{~cm}$ long) is used as a concentrated solar energy absorber and in the same time as a transfer wall to the powder. The temperature profile at the cylinder surface is directly depending on the incident solar illumination inside the cavity. At a wall mean temperature of $1000^{\circ} \mathrm{C}$ the temperature difference does not exceed $100^{\circ} \mathrm{C}$. The mean residence time of particles in the reactor varies from 1 to $2 \mathrm{~min}$. when the mass flow rate varies from 5 to $25 \mathrm{~g}$. $\mathrm{min}^{-1}$ and rotation from 8 to $2 \mathrm{rpm}$.
\end{abstract}

\section{Nomenclature}

$C_{\mathrm{p}} \quad$ : Chaleur spécifique du produit.

$D$ : Diamètre de la paroi de transfert.

$E_{\mathrm{s}}^{0} \quad$ : Eclairement solaire incident.

$L \quad$ : Longueur de la paroi.

$\dot{m} \quad$ : Débit massique du produit.

$S \quad$ : Surface équivalente à la partie éclairée du concentrateur.

$T$ : Température de la paroi de transfert.

$W_{\text {ex }}$ : Puissance évacuée par le réacteur.

$W_{\text {th }}$ : Puissance absorbée par le produit à traiter.

$W_{\mathrm{u}}$ : Puissance absorbée par la paroi de transfert.

$x$ : Demi-distance entre les bords des deux écrans de l'installation solaire.

$\alpha \quad$ : Coefficient caractéristique du réacteur appliqué au calcul de $W_{\text {ex }}$.

$\gamma \quad$ : Angle d'inclinaison du réacteur.

$\omega$ : Vitesse de rotation du réacteur.

$\eta \quad$ : Rendement énergétique de l'installation solaire (concentrateur + paroi de transfert).

$\rho_{\mathrm{a}} \quad$ : Densité apparente du produit. $\tau \quad:$ Taux de remplissage du réacteur.

$\Delta H_{T}$ : Enthalpie totale, somme de la chaleur sensible et de l'enthalpie de la réaction.

$\Delta H_{T}^{0}$ : Enthalpie de la réaction à $T$.

$\psi \quad$ : Rendement thermochimique du réacteur.

1. Introduction - L'utilisation rationnelle de l'énergie solaire sous sa forme thermique, par conséquent sans transformation préalable, peut s'appliquer aux domaines de la thermochimie suivants :

- la minérallurgie qui vise à valoriser les matières premières par simples traitements thermiques conduisant à la transformation et (ou) à la séparation de phases condensées ou gazeuses à plus grande valeur ajoutée,

- le stockage chimique de l'énergie thermique au moyen d'opérations réversibles (cycles thermochimiques, stockage saisonnier),

- la production de vecteurs énergétiques $\left(\mathrm{H}_{2}, \mathrm{CO}\right.$, décomposition thermique de la biomasse). 
Ces opérations s'effectuent généralement dans des domaines de températures qui, pour des raisons technologiques et économiques, doivent se situer audessous de la barrière $T=1200^{\circ} \mathrm{C}$.

De telles réactions thermochimiques s'envisagent dans la perspective de mise au point de procédés industriels simples, fiables, s'opérant le plus généralement dans l'air. Parmi eux la valorisation par décomposition de matériaux naturels (décarbonatation, déshydratation) constitue un domaine d'application de l'ênergie solaire concentrée particulièrement intéressant mais implique le développement de dispositifs adaptés [1-3]. D'où notre objectif de mettre au point et de caractériser un réacteur à lit brassé et un réacteur à-lit fluidisé annulaire [4]. Nous présentons dans ce qui suit les résultats obtenus avec le réacteur à lit brassé.

2. Dispositif expérimental. - Le dispositif expérimental est représenté dans son ensemble par la photo sur laquelle apparaissent :

- le dispositif optique horizontal de concentration $\mathrm{du}$ rayonnement solaire,

- le réacteur proprement dit, développé autour d'une paroi de transfert (Fig. 1) quasi totalement absorbante et chargée de transmettre l'énergie aux particules.

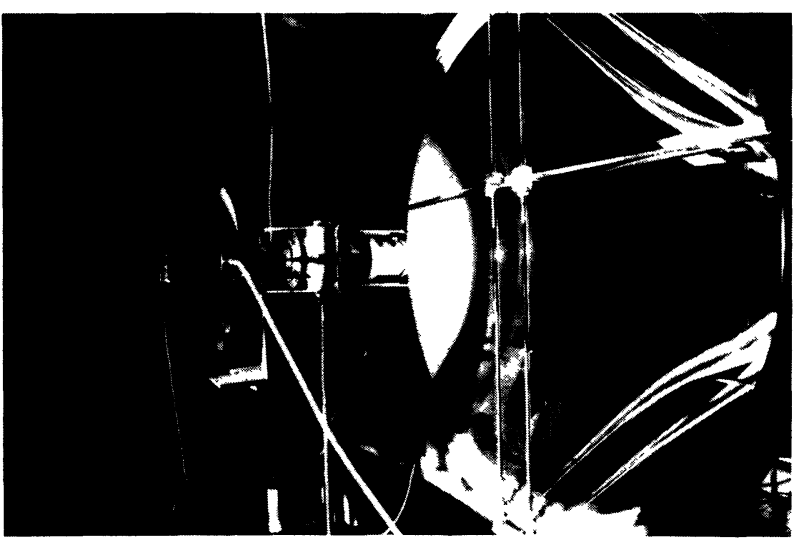

Photo 1.

2.1 L'installation SOlaire. - Celle-ci comprend un héliostat orientable et un concentrateur parabolique de caractéristiques suivantes :

- diamètre : $2 \mathrm{~m}$

- distance focale : $0,85 \mathrm{~m}$

- angle d'ouverture : $120^{\circ}$

- taux de concentration : 16000 .

La puissance maximum disponible au foyer d'une telle installation est de $1700 \mathrm{~W}$ pour un éclairement incident de $1 \mathrm{~kW} / \mathrm{m}^{2}$. A l'aide de deux écrans coulissants une partie de la surface du miroir parabolique peut être occultée permettant ainsi de moduler la puissance disponible au foyer.



Fig. 1. - Représentation schématique de l'installation solaire : 1. Ecran mobile; 2. Concentrateur parabolique; 3. Vers l'héliostat; 4. Rayonnement solaire concentré; 5. Cavité absorbante (paroi de transfert); 6. Rayonnement incident.

[Schematic view of the solar equipment : 1 . Moving screen; 2. Parabolic concentrator; 3 . To heliostat; 4. Concentrated solar rays; 5 . Absorbing cavity (transfer wall); 6 . Incident solar rays.]

2.2 LA PAROI DE TRANSFERT. - Ce récepteur est une cavité cylindrique en acier réfractaire au $\mathrm{Ni}-\mathrm{Cr}$ $(25 \%-20 \%)$ résistant à des températures élevées $\left(1150^{\circ} \mathrm{C}\right)$ en milieu oxydant et de conductivité thermique $\lambda \simeq 25 \mathrm{~W} / \mathrm{m} .{ }^{\circ} \mathrm{C}$ à $T=900^{\circ} \mathrm{C}$. Ce tube cylindrique est en partie fermé sur la face postérieure. Un diaphragme circulaire disposé sur la face antérieure recevant le flux lumineux limite les pertes par rayonnement de la capacité.

2.3 Le RÉACTEUR. - La figure 2 schématise le réacteur proprement dit caractérisé pour l'essentiel par :

- un système d'alimentation des poudres par vis d'Archimède montée dans l'axe du récepteur et coaxialement à un axe creux central susceptible d'évacuer les gaz issus d'une réaction en phase hétérogène. Ce système joue le rôle d'échangeur d'énergie entre gaz et particules,

- un ensemble de moto-réducteurs qui assure la mise en rotation séparément de la cavité et de la vis d'Archimède. La vitesse de rotation du four est directement reliée à la tension d'alimentation du moteur,

- une protection isolante radiale, en laine d'alumine, et axiale par des pièces en asbestex,

- une série de thermocouples le long d'une génératrice de la cavité cylindrique dont les extrémités sont positionnées dans l'épaisseur du métal à $0,1 \mathrm{~mm}$ près de la paroi interne du récepteur. Les signaux de ces sondes sont transmis à une centrale de mesure par l'intermédiaire d'un stator et d'un rotor solidaire du mouvement d'entraînement du réacteur.

3. Détermination des temps de séjour. - Le temps de séjour d'une particule dans le réacteur à lit brassé est un paramètre déterminant dans la recherche d'un optimum de fonctionnement. 


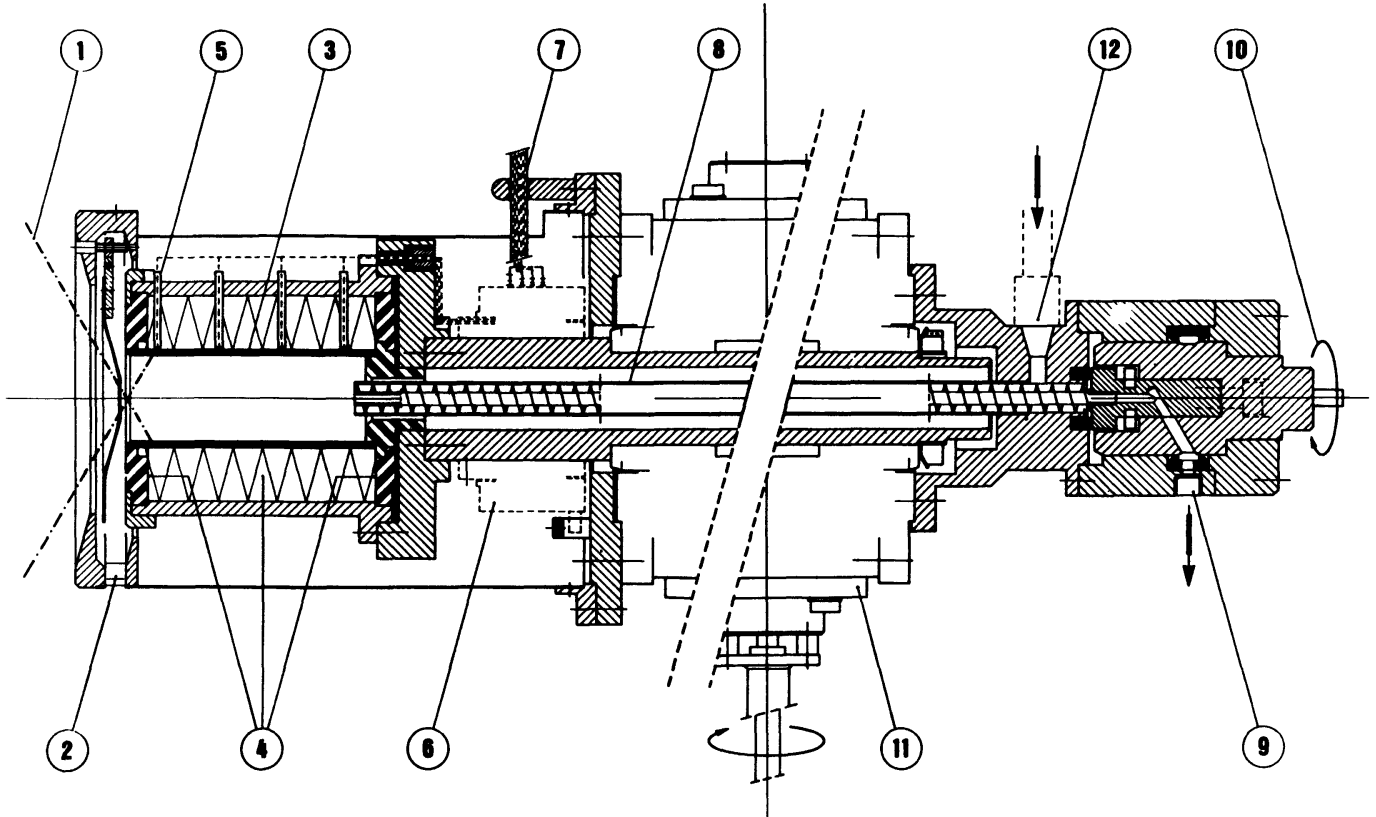

Fig. 2. - Schéma du réacteur à lit brassé : 1. Rayonnement solaire concentré; 2. Sortie des poudres; 3. Paroi de transfert; 4. Isolants thermiques; 5 . Thermocouples; 6. Collecteur de mesures tournant; 7. Centrale de mesures; 8 . Vis sans fin; 9. Aspiration de gaz; 10. Rotation de la vis sans fin; 11. Dispositif de mise en rotation du réacteur; 12. Entrée des produits.

[Schematic of the rotary kiln : 1. Concentrated solar rays; 2. Powder outlet; 3. Metallic transfer wall; 4. Insulators; 5. Thermocouples; 6. Rotating collector for temperature measurements; 7. Recording device; 8. Endless screw; 9. Gas exhaust ; 10. Endless screw rotation; 11. Device for reactor rotation; 12. Powder inlet.]

Ce paramètre dépend :

- de facteurs de configuration : diamètre et longueur de la capacité, angle d'inclinaison du four et nature de la paroi (rugosité),

- et de facteurs de fonctionnement : pour l'essentiel la vitesse de rotation de l'installation et le débit massique du produit.

Ces facteurs étant fixés a priori, le transfert de la charge s'effectue par brassage continu de chaque particule au sein de leur ensemble constitué par le talus. Le comportement macroscopique de ce dernier varie selon les conditions opératoires d'un régime périodique dit de slumping à très faible vitesse à un mode d'écoulement régulier de la charge [5]. Les divers modes d'avancement de la charge dans notre réacteur sont présentés sur la figure 3 .

Le talus dans ces mêmes conditions se caractérise alors par un angle de glissement et une hauteur maximum constants (Fig. 3).

La détermination des temps de séjour moyens à partir des caractéristiques ci-dessus a fait l'objet de nombreuses relations empiriques (cf. Annexe). Nous nous sommes attachés pour notre part à la mesure de la répartition des temps de séjour des particules dans le réacteur.

3.1 MéTHOde EXPÉRIMENTALE. - La méthode consiste à définir la réponse $F(t)$ en fonction du temps à un signal échelon introduit dans le système. Le signal échelon est provoqué par l'admission dans le réacteur
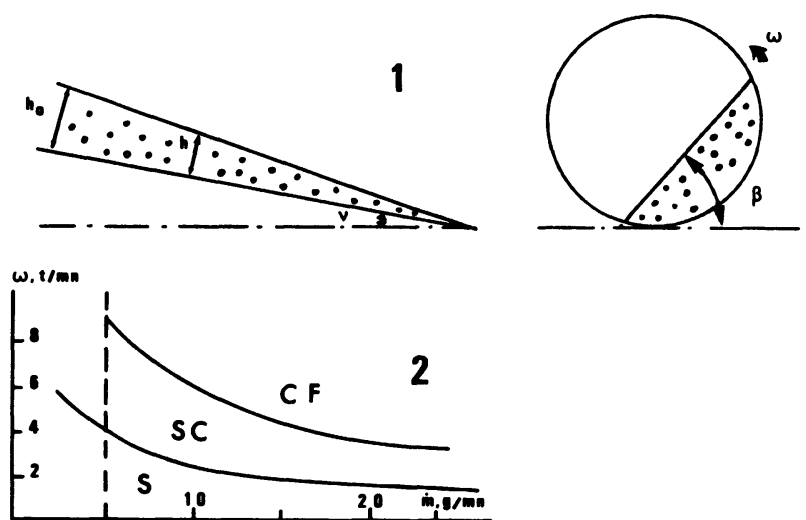

Fig. 3. - Déplacement de la charge : 1. Géométrie du talus : $h_{0}$ épaisseur maximum, $\beta$ angle d'inclinaison -2 . Modes de déplacement : $S$ "slumping ", $C F$ régime permanent, $\mathrm{SC}$ régime intermédiaire.

[Load displacement : 1 . Slope geometry $: h_{0}$ maximum depth, $\beta$ angle of repose -2 . Displacement modes : $S$ "slumping ", CF continuous flow, SC intermediate running.]

à $t=0$ d'un produit $\mathrm{B}$ de densité et granulométrie comparables à celles du produit $A$ initial. Les matériaux $A$ et $B$ se différencient toutefois par une propriété physique susceptible de varier de $\omega_{0}^{-}$à $\omega_{0}^{+}$proportionnellement à la concentration du mélange :

$$
\alpha . A+(1-\alpha) B \text {. }
$$


Nous avons choisi des matériaux dont les coefficients de réflexion sont suffisamment différents pour permettre de suivre l'évolution de cette propriété en fonction de la concentration du mélange. Les produits issus du réacteur sont recueillis sur une piste circulaire animée d'un mouvement de rotation uniforme (Fig. 4).

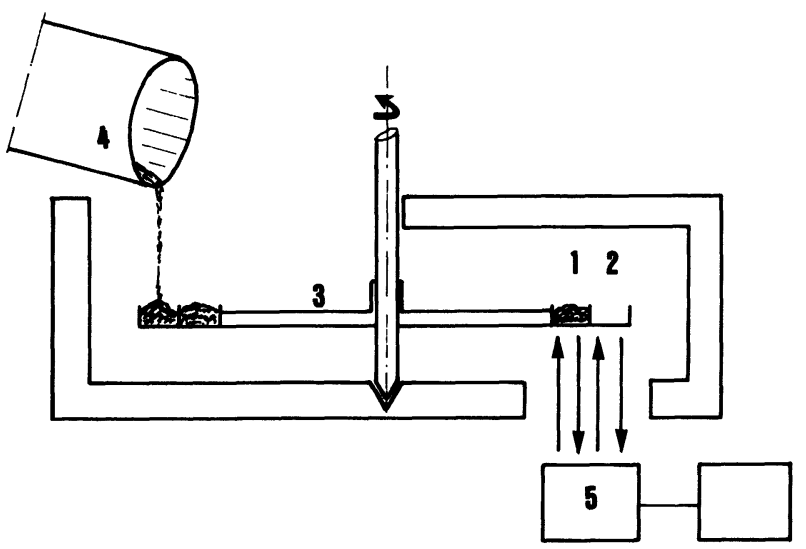

Fig. 4. - Schéma du dispositif utilisé pour la détermination de la répartition des temps de séjour : 1 . Référence; 2. Echantillon; 3. Disque tournant ; 4. Réacteur; 5. Spectrophotomètre.

[Schematic of the device used to determine residence time distribution : 1. Reference; 2. Sample; 3. Rotating disk; 4. Reactor; 5. Spectrophotometer.]
L'analyse en continu, à l'aide d'un spectrophotomètre, du cordon de poudre ainsi obtenu donne la variation du coefficient de réflexion du mélange en fonction du temps. Un étalonnage préalable du système permet d'en déduire l'évolution de la concentration.

3.2 Résultats. - Les essais ont été effectués sur la calcite et des phosphates à gangue carbonatée, pour diverses valeurs de débit massique et de vitesse de rotation du réacteur. Dans les deux cas le signal échelon est provoqué par l'introduction d'ilménite dans le réacteur. La série de courbes expérimentales (Fig. 5) donnant les réponses au signal échelon, montre que le fonctionnement de notre réacteur est proche de celui d'un réacteur piston. Les tableaux I et II rassemblent les résultats expérimentaux obtenus et permettent de comparer les valeurs des temps de séjour moyens mesurés et calculés à partir de différents modèles [5-7] (Annexe). On constate que pour nos conditions opératoires, le meilleur accord est obtenu à partir d'une relation du type de celle de Wahl [8] :

$$
\bar{t}=C\left(\frac{L}{D}\right)^{2}\left(\frac{D}{h_{0}}\right)^{0,6} \frac{\operatorname{tg} \beta}{\omega}
$$

$C$ est une constante déterminée empiriquement.

Nous n'avons pas observé de variation notable du temps de séjour en fonction de la granulométrie $(100-315 \mu \mathrm{m})$ des poudres utilisées.

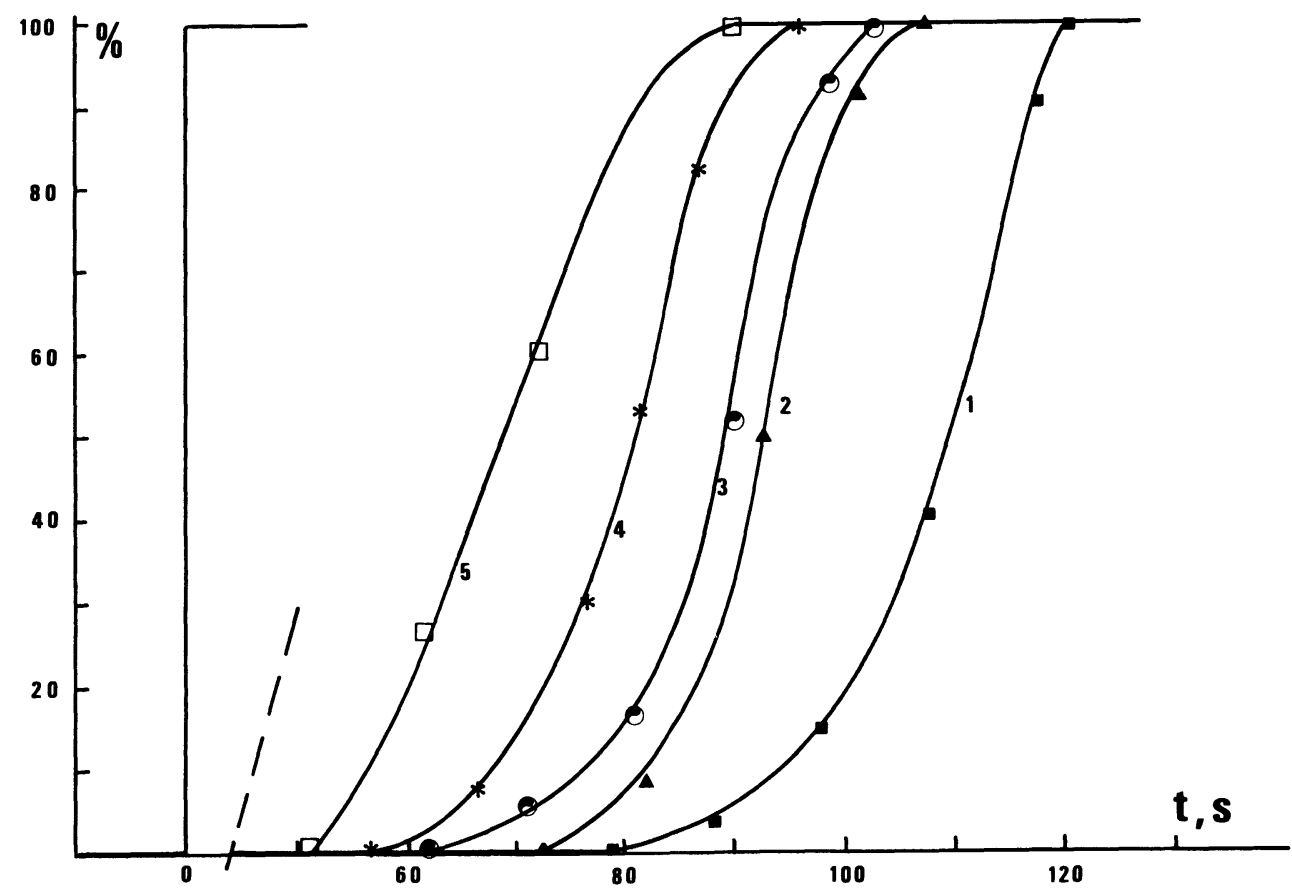

Fig. 5. - Courbes de réponse à un signal échelon : $\omega=6 \mathrm{t} . \mathrm{min}^{-1}: 1.5 \mathrm{~g} \cdot \mathrm{min}^{-1} ; 2.10 \mathrm{~g} \cdot \mathrm{min}^{-1} ; 3.15 \mathrm{~g} \cdot \mathrm{min}^{-1} ; 4$. 20 g.min. ${ }^{-1} ; 5.25$ g. min. ${ }^{-1}$.

[Response curves to a step signal : $\omega=6 \mathrm{rpm}: 1.5 \mathrm{~g} \cdot \mathrm{min}^{-1} ; 2.10 \mathrm{~g} \cdot \mathrm{min}^{-1} ; 3.15 \mathrm{~g} \cdot \mathrm{min} .^{-1} ; 4.20 \mathrm{~g} \cdot \mathrm{min} \cdot{ }^{-1}$; 5. 25 g. min. ${ }^{-1}$.] 
Tableau I. - Temps de séjour moyen $\bar{t}$ des particules de calcite $\left(\left(^{*}\right)\right.$ 200-315 $\mu \mathrm{m}$ et $\left({ }^{* *}\right)$ 100-200 $\left.\mu \mathrm{m}\right)$ dans le réacteur $\left(\varnothing=4,2 \mathrm{~cm}, L=12 \mathrm{~cm}, \gamma=2^{\circ}\right)$. Comparaison des valeurs expérimentales et calculées.

[Mean residence time $\bar{t}$ of calcite particles $\left(\left(^{*}\right) 200-315 \mu \mathrm{m}\right.$ and $\left.\left(^{* *}\right) 100-200 \mu \mathrm{m}\right)$ in the reactor $\varnothing=4.2 \mathrm{~cm}$, $L=12 \mathrm{~cm}, \gamma=2^{\circ}$ ). Experimental and calculated values.]

\begin{tabular}{|l|c|c|c|c|c|c|c|}
\hline$\omega \mathrm{t} / \mathrm{min}$. & $\dot{m} \mathrm{~g} / \mathrm{min}$. & $\beta^{0}$ & $h^{0} \mathrm{~mm}$ & $\bar{t}$ min. & Sullivan [5] & Heilig. [6] & $\begin{array}{c}\text { Wahl [8] } \\
C=0,444\end{array}$ \\
\hline $2\left(^{*}\right)$ & 5 & 35 & 7 & 3,75 & 7,48 & 7,45 & 3,72 \\
2 & 10 & 35 & 10 & 2,33 & & & 3 \\
2 & 15 & 35 & 14 & 2,50 & & & 2,45 \\
2 & 20 & 35 & 17 & 2,00 & & & 2,18 \\
\hline $4\left(^{*}\right)$ & 5 & 35 & 6 & 2,08 & 3,74 & 3,73 & 2,04 \\
4 & 10 & 35 & 8 & 1,47 & & & 1,72 \\
4 & 15 & 35 & 12 & 1,23 & & & 1,35 \\
4 & 20 & 35 & 15 & 1,12 & & & 1,18 \\
\hline $6\left(^{*}\right)$ & 5 & 35 & 5 & 1,58 & 2,49 & 2,48 & 1,52 \\
6 & 7 & 35 & 6 & 1,12 & & & 1,36 \\
6 & 10 & 35 & 9 & 1,5 & & & 1,07 \\
6 & 15 & 35 & 13 & 0,75 & & & 0,85 \\
\hline $8\left(^{*}\right)$ & 5 & 35 & 4,5 & 1 & 1,87 & 1,86 & 1,21 \\
8 & 7 & 35 & 5,5 & 1 & & & 1,07 \\
8 & 10 & 35 & 6,5 & 0,67 & & & 0,97 \\
8 & 15 & 35 & 9 & 0,83 & & & 0,80 \\
\hline $4\left(^{* *}\right)$ & 5 & 35 & 5,5 & 2,17 & 3,74 & 3,73 & 2,15 \\
4 & 7 & 35 & 7 & 1,83 & & & 1,86 \\
4 & 10 & 35 & 10 & 1,33 & & & 1,50 \\
4 & 15 & 35 & 14 & 1,22 & & & 1,23 \\
\hline $6\left(^{* *}\right)$ & 5 & 35 & 5,5 & 1,33 & 2,49 & 2,48 & 1,43 \\
6 & 7 & 35 & 7 & 1,08 & & & 1,24 \\
6 & 10 & 35 & 9,5 & 1,17 & & & 0,90 \\
6 & 15 & 35 & 12 & 1 & & & \\
\hline
\end{tabular}

4. Caractérisations thermique et énergétique. 4.1 RENDEMENT DE L'INSTALLATION SOLAiRE. - La puissance $W$ disponible au foyer est mesurée à l'aide d'un calorimètre de géométrie identique à celle de la paroi de transfert du réacteur. Un pyrhéliomètre mesure l'éclairement solaire incident $E_{\mathrm{s}}^{0}$ qui est modulable à l'aide d'écrans mobiles (Fig. 1).

Le rendement de l'installation est défini par le rapport :

$$
\eta=\frac{W_{\mathrm{u}}}{E_{\mathrm{s}}^{0} S(x)}
$$

où $S(x)$ est la surface du concentrateur utilisé en fonction de l'ouverture $x$ des écrans mobiles.
Ce rendement est bas pour de faibles ouvertures du fait de l'importance des ombres portées. Il augmente avec l'ouverture jusqu'à une valeur de 0,50 (Fig. 6) proche du rendement du four solaire utilisé $(0,55)$. Ces résultats indiquent que la géométrie de notre réacteur est bien adaptée, le coefficient d'absorption de la cavité étant voisin de l'unité.

4.2 RÉPARTITION DU FLUX À LA PAROI. - La répartition du flux le long d'une génératrice du cylindre constituant la paroi de transfert peut être définie expérimentalement ou par le calcul $[9,10]$ à partir des caractéristiques géométriques du concentrateur et du récepteur.

La figure 7 traduit l'éclairement théorique à la paroi 
Tableau II. - Temps de séjour moyen $\bar{t}$ des particules de minerais de phosphates $(100-200 \mu \mathrm{m})$ dans le réacteur $\left(\varnothing=5,2 \mathrm{~cm}, L=12 \mathrm{~cm}, \gamma=2^{\circ}\right)$. Comparaison des valeurs expérimentales et calculées.

[Mean residence time $\bar{t}$ for phosphate rock particles $(100-200 \mu \mathrm{m})$ in the reactor $(\varnothing=5.2 \mathrm{~cm}, L=12 \mathrm{~cm}$, $\gamma=2^{\circ}$ ). Experimental and calculated values.]

\begin{tabular}{|c|c|c|c|c|c|c|c|}
\hline$\omega \mathrm{t} / \mathrm{min}$. & $\dot{m} \mathrm{~g} / \mathrm{min}$. & $\beta^{0}$ & $h^{0} \mathrm{~mm}$ & $\bar{t}$ min. & Sullivan [5] & Heilig. [6] & $\begin{array}{c}\text { Wahl }[8] \\
C=0,654\end{array}$ \\
\hline 4 & 5 & $45-40$ & 7 & 2,15 & $3,39-3,20$ & $3,68-3,34$ & $2,86-2,40$ \\
4 & 10 & $45-40$ & 7 & 2,55 & $3,39-3,20$ & $3,68-3,34$ & $2,86-2,40$ \\
4 & 15 & $45-40$ & 10 & 1,70 & $3,39-3,20$ & $3,68-3,34$ & $2,31-1,94$ \\
4 & 20 & $45-40$ & 12 & 2,27 & $3,39-3,20$ & $3,68-3,34$ & $2,07-1,74$ \\
4 & 25 & $40-35$ & 14 & 1,53 & 3,20 & 3,34 & 1,59 \\
\hline 6 & 5 & 40 & 5,5 & 1,83 & 2,13 & 2,23 & 1,85 \\
6 & 10 & $40-35$ & 6,5 & 1,53 & & & 1,67 \\
6 & 15 & $40-35$ & 8 & 1,45 & & & 1,48 \\
6 & 20 & 40 & 10 & 1,32 & & & 1,29 \\
6 & 25 & 40 & 13 & 1,18 & & & 1,10 \\
\hline 8 & 5 & 40 & 5 & 1,58 & 1,60 & 1,67 & 1,47 \\
8 & 10 & 40 & 6 & 1,33 & & & 1,32 \\
8 & 15 & 40 & 8 & 1,08 & & & 1,11 \\
8 & 20 & 40 & 9 & 1,05 & & & 1,03 \\
8 & 25 & 40 & 10 & 0,95 & & & 0,97 \\
\hline 10 & 5 & 40 & 5 & 1,12 & 1,28 & 1,34 & 1,18 \\
10 & 10 & 40 & 6 & 1,05 & & & 1,05 \\
10 & 15 & 40 & $7-7,5$ & 0,92 & & & 0,94 \\
10 & 20 & $40-38$ & 8,5 & 0,83 & & & 0,86 \\
10 & 25 & 40 & 10 & 0,78 & & & 0,78 \\
\hline 12 & 5 & 40 & 4 & 1,08 & 1,07 & 1,11 & 1,12 \\
12 & 10 & 40 & 5 & 0,88 & & & 0,98 \\
12 & 15 & 40 & 6,5 & 0,89 & & & 0,77 \\
12 & 20 & 40 & 7,5 & 0,68 & & & 0,69 \\
12 & 25 & $40-35$ & 9 & 0,75 & & & \\
\hline
\end{tabular}

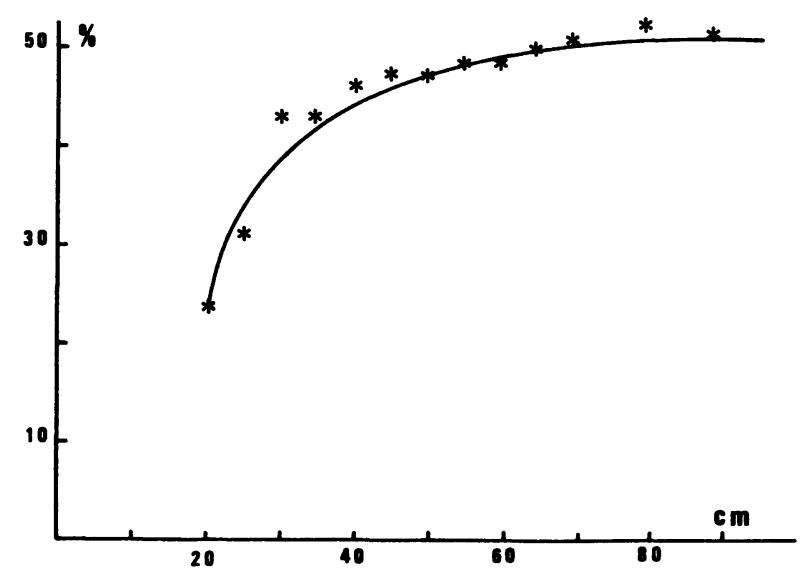

Fig. 6. - Rendement de l'installation solaire en fonction de la demi-distance entre les écrans mobiles.

[Solar-equipment yield versus half-distance between moving screens.] pour notre système. On note un gradient très important avec une forte concentration d'énergie dans les premiers centimètres du collecteur. Ce phénomène, dû aux caractéristiques optiques du concentrateur, n'est pas très favorable à l'obtention d'une température homogène à la paroi. Des systèmes à plus faible ouverture et concentration sembleraient mieux adaptés pour des réactions isothermes.

4.3 RÉPARTITION DES TEMPÉRATURES. - Les températures sont mesurées, à l'équilibre thermique de la paroi, à l'aide de thermocouples et par pyrométrie optique.

4.3.1 Gradients axiaux. - L'ensemble des profils de température obtenus expérimentalement pour plusieurs puissances incidentes est représenté sur la figure 8. Il apparaît que :

- le gradient de température est élevé : les échanges 


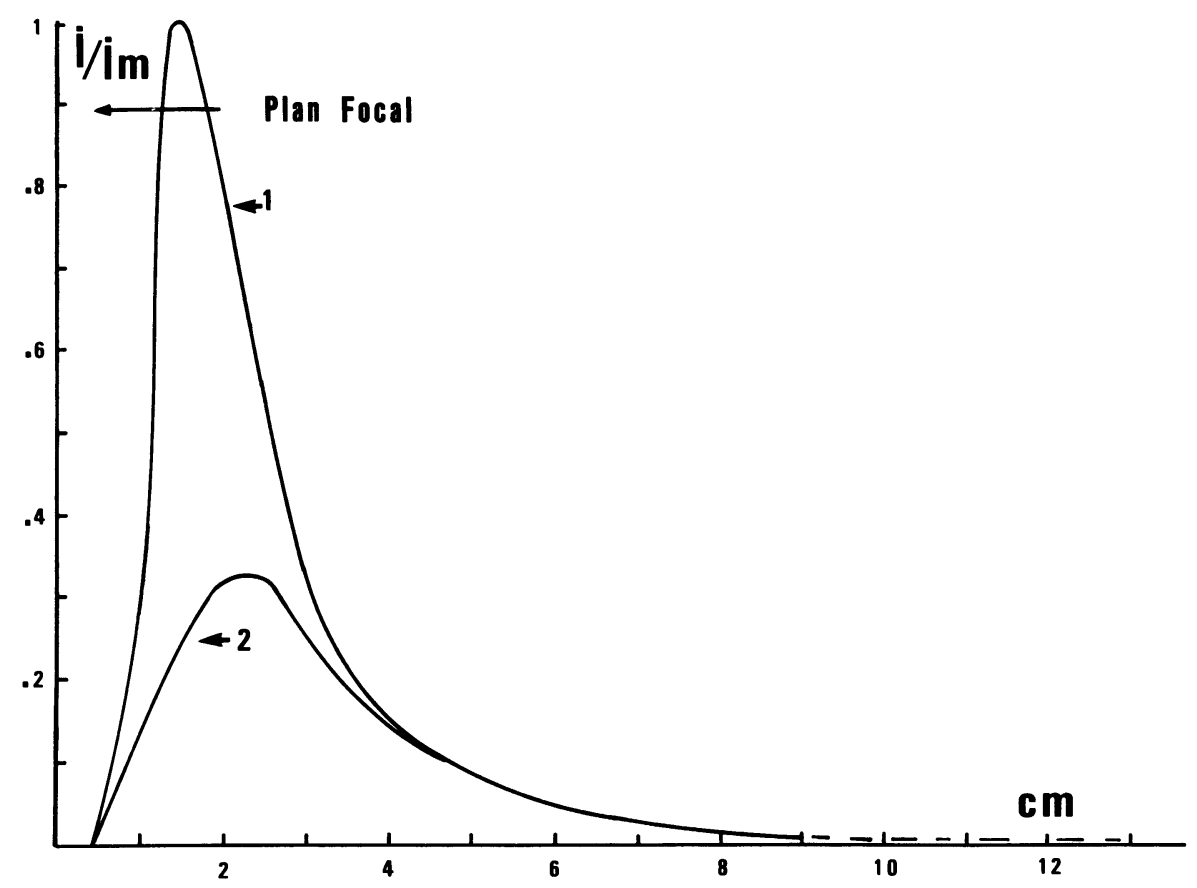

Fig. 7. - Profils de l'éclairement solaire incident dans la cavité $\left(I_{\mathrm{m}}=55 \mathrm{~W} . \mathrm{cm}^{-2}\right): 1$. A pleine ouverture des écrans; 2. Distance des écrans $=80 \mathrm{~cm}$.

[Incident solar illumination profiles inside the cavity $\left(I_{\mathrm{m}}=55 \mathrm{~W} . \mathrm{cm}^{-2}\right): 1$. Full aperture of the screens $(200 \mathrm{~cm}) ; 2$. For a distance of $80 \mathrm{~cm}$ between screens.]

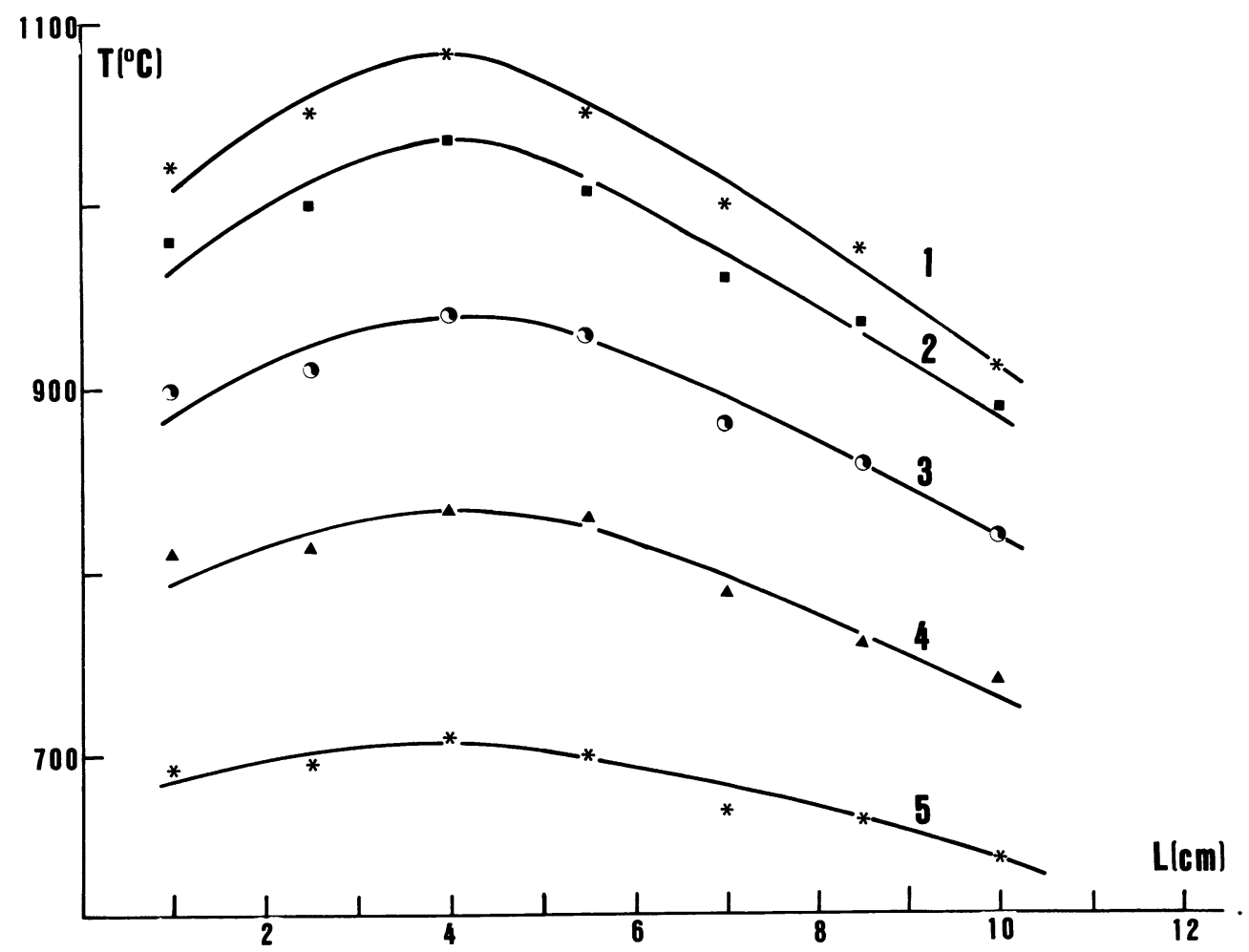

Fig. 8. - Profil des températures à la paroi $(\varnothing=4,2 \mathrm{~cm}, L=12 \mathrm{~cm})$ du réacteur pour diverses puissances incidentes : 1. $W_{\mathrm{u}}=767 \mathrm{~W} ; 2 . W_{\mathrm{u}}=691 \mathrm{~W} ; 3 . W_{\mathrm{u}}=560 \mathrm{~W} ; 4 . W_{\mathrm{u}}=497 \mathrm{~W} ; 5 . W_{\mathrm{u}}=294 \mathrm{~W}$.

[Reactor wall $(\varnothing=4.2 \mathrm{~cm}, L=12 \mathrm{~cm})$ temperature profiles for various incident solar power : $1 . W_{\mathrm{u}}=767 \mathrm{~W} ; 2 . W_{\mathrm{u}}=$ $691 \mathrm{~W} ; 3 . W_{\mathrm{u}}=650 \mathrm{~W} ; 4 . W_{\mathrm{u}}=497 \mathrm{~W} ; 5 . W_{\mathrm{u}}=294 \mathrm{~W}$. $]$ 
thermiques par rayonnement et conduction ne permettent pas d'homogénéiser la température,

- ce gradient augmente avec la puissance incidente : des travaux en cours montrent que cette évolution est due à la répartition du flux incident à la paroi.

En conséquence, pour des réactions qui sont envisagées (décarbonatation à $800-900^{\circ} \mathrm{C}$ ) la longueur des collecteurs devra être limitée à $12 \mathrm{~cm}$.

4.3.2 Gradients radiaux. - Des mesures pyrométriques effectuées sur des trous borgnes, de différentes profondeurs, n'ont pas permis de mettre en évidence de gradient notable dans l'épaisseur $(3 \mathrm{~mm})$ de la paroi. De ce fait, les échanges paroi-particule pourront s'effectuer dans de bonnes conditions aussi bien à la surface interne qu'à la surface externe (cas du réacteur à lit fluidisé annulaire) [4].

La comparaison des mesures thermométriques et pyrométriques permet l'estimation du coefficient d'émission monochromatique de la paroi oxydée $\left(\varepsilon_{\lambda}=0,8\right.$ pour $\left.\lambda=0,6328 \mu \mathrm{m}\right)$.

5. Bilan thermique global. Optimisation du réacteur. - Considérons une réaction thermochimique effectuée au sein du réacteur à lit brassé et nécessitant une puissance

$$
W_{\mathrm{th}}=\dot{m} \Delta H_{T}=\dot{m} \rho_{\mathrm{a}} \Delta H_{T}^{0}+\dot{m} C_{\mathrm{p}} \Delta T .
$$

Le réacteur lui-même absorbe pour maintenir son équilibre thermique une puissance $W_{\mathrm{ex}}$. Des mesures ont montré que cette puissance est [3] une fonction linéaire de la surface interne du réacteur pour une température moyenne donnée : $W_{\mathrm{ex}}=\alpha \pi D L$. La puissance reçue par la paroi s'écrit :

$$
W_{\mathrm{u}}=W_{\mathrm{th}}+W_{\mathrm{ex}} .
$$

L'objectif de cette approche est d'établir une corrélation entre les paramètres énergétiques $\left(W_{\mathrm{u}}, W_{\mathrm{th}}, \Delta H_{T}\right)$, géométriques $(D$ et $L)$ et de fonctionnement $\left(\dot{m}, \rho_{\mathrm{a}}\right.$, $\tau(\omega), \bar{t}(\omega))$.

Le débit massique s'exprime par :

$$
\dot{m}=\frac{\pi D^{2} L \tau \rho_{\mathrm{a}}}{4 \bar{t}},
$$

il vient :

$$
W_{\mathrm{th}}=\frac{\pi D^{2} L \tau \rho_{\mathrm{a}}}{4 \bar{t}} \Delta H_{\mathrm{T}},
$$

et

$$
L=\frac{W_{\mathrm{u}}}{\alpha \pi D+\frac{\tau}{4 \bar{t}} \pi D^{2} \rho_{\mathrm{a}} \Delta H_{T}} .
$$

Cette dernière équation conduit à calculer la longueur minimum de la paroi de transfert ou le débit massique maximum pour une décarbonatation totale.

Nous présentons dans le tableau III les résultats du calcul pour une décarbonatation totale de calcite avec :

$$
\begin{aligned}
\rho_{\mathrm{a}} & =1 \mathrm{~g} \cdot \mathrm{cm}^{-3} ; \tau=15 \% ; \bar{t}=60 \text { et } 120 \mathrm{~s} ; \\
\Delta H_{T} & =2538 \mathrm{~J} \cdot \mathrm{g}^{-1}\left(T=850^{\circ} \mathrm{C}\right) ; \alpha=4,5 \mathrm{~W} . \mathrm{cm}^{-2} .
\end{aligned}
$$

Les rendements thermochimiques $\psi=W_{\mathrm{th}} / W_{\mathrm{u}}$ sont nettement supérieurs pour les longueurs les plus faibles. En effet, les pertes d'énergie $\left(W_{\mathrm{ex}}\right)$ sont alors réduites. Il convient néanmoins de considérer que la cinétique de décarbonatation d'un grain dans les conditions

\begin{tabular}{|c|c|c|c|c|c|c|c|c|}
\hline \multirow[b]{2}{*}{ Série } & \multirow[b]{2}{*}{$D(\mathrm{~cm})$} & \multirow[b]{2}{*}{$\tau \%$} & \multirow[b]{2}{*}{$\bar{t}(\mathrm{~s})$} & \multicolumn{2}{|c|}{$W_{\mathrm{u}}=1400 \mathrm{~W}$} & \multirow[b]{2}{*}{$L(\mathrm{~cm})$} & \multirow[b]{2}{*}{$\dot{m}\left(\mathrm{~g} \cdot \mathrm{s}^{-1}\right)$} & \multirow[b]{2}{*}{$\psi \%$} \\
\hline & & & & $W_{\text {ex }}(\mathrm{W})$ & $W_{\text {th }}(\mathrm{W})$ & & & \\
\hline \multirow{2}{*}{ I } & \multirow{2}{*}{5,25} & \multirow{2}{*}{15} & 60 & 490 & 910 & 6,6 & 0,357 & 65 \\
\hline & & & 120 & 727 & 673 & 9,8 & 0,265 & 48 \\
\hline \multirow{2}{*}{ II } & \multirow{2}{*}{4,25} & \multirow{2}{*}{15} & 60 & 558 & 842 & 9,3 & 0,330 & 60 \\
\hline & & & 120 & 799 & 601 & 13,3 & 0,235 & 43 \\
\hline \multirow{2}{*}{ III } & \multirow{2}{*}{3,60} & \multirow{2}{*}{15} & 60 & 618 & 782 & 12,1 & 0,308 & 56 \\
\hline & & & 120 & 857 & 543 & 16,8 & 0,214 & 39 \\
\hline
\end{tabular}
opératoires envisagées implique des temps de séjour moyens de l'ordre de $2 \mathrm{~min}$. Ainsi il apparaît que pour optimiser le rendement thermochimique compte tenu de la cinétique de décarbonatation, les dimensions

Tableau III. - Conditions opératoires calculées à partir du bilan global d'énergie.

[Operating conditions calculated from total energy balance.] 
optimales de la paroi devront se situer entre 10 et $12 \mathrm{~cm}$ pour des diamètres voisins de $5 \mathrm{~cm}$.

6. Conclusion. - Nous avons réalisé un réacteur à lit brassé adapté à une installation solaire $(2 \mathrm{~kW})$ à forte concentration. Son comportement thermique et dynamique a été caractérisé par des mesures de profils de température à la paroi, de répartition des temps de séjour des particules et du mode d'écoulement de la charge. Ces données nous permettent d'optimiser le réacteur pour une réaction donnée. Les premiers résultats obtenus sur la calcite (décarbonatation) sont en bon accord avec les prévisions. Le réacteur apparaît bien adapté pour ce type d'application. En ce qui concerne l'installation solaire des systèmes à plus faibles ouverture et concentration seraient mieux appropriés.
Annexe. - DÉTERMINATION DES TEMPS DE SÉJOUR MOYEN PAR DES RELATIONS EMPIRIQUES.

1) $\bar{t}=\frac{1,77 L \sqrt{\beta}}{\gamma D \omega}$ d'après Sullivan [5].

2) $\bar{t}=\frac{0,318 . L}{\omega \operatorname{tg} \alpha D}$ d'après Heiligenstaedt [6] où $\alpha$ est l'angle de l'hélice défini par

$$
\sin \alpha=\frac{\sin \gamma}{\sin \beta}
$$

3) $\bar{t}=\frac{0,035(\beta+24) \cdot L}{D \omega \gamma}$ d'après Bayard [7].

4) Dans le cas de fours horizontaux $(\gamma=0)$

$$
\bar{t}=0,91\left(\frac{L}{D}\right)^{2} \cdot\left(\frac{D}{h_{0}}\right)^{0,6} \frac{\operatorname{tg} \beta}{\omega} \text { d'après Wahl [8] }
$$

\section{Bibliographie}

[1] Flamant, G., Thèse de Docteur Ingénieur. Université Paul Sabatier, Toulouse, 1978.

[2] Villermaux, J., Entropie 85 (1979) 25-31.

[3] Badie, J. M., Bonet, C., Faure, M., Flamant, G., Foro, R. et Hernandez, D., Chem. Eng. Sci. 35 (1980) 413-420.

[4] FORO, R., Thèse de Docteur Ingénieur. Université de Perpignan, 1981.

[5] Sullivan, J. D., Passage of solid particles through rotary cylindrical kilns, Tech. Pap. 384. Government Printing Office (1927).
[6] Heiligenstaedt, W., Thermique appliquée aux fours industriels (Dunod) 1971.

[7] Bayard, R. A., Chem. Metall. Eng. 52, no 3 (1945) 100-102.

[8] WaHL, L., Chem. Eng. Sci. 1, no 6 (1952) 253-258.

[9] Alcayaga, O., Thèse de 3e Cycle. Université de Poitiers, 1977.

[10] Kamada, O., Sol. Energy 9, no 1 (1965) 39-47. 